\title{
Shear Stress Intensity Factors for a Planar Crack With Slightly Curved Front
}

\author{
Huajian Gao \\ James R. Rice \\ Division of Applied Sciences, \\ Harvard University, \\ Cambridge, Mass. 02138
}

Recent work (Rice, 1985a) has presented the calculations of the first order variation in an elastic displacement field associated with arbitrary incremental planar advance of the location of the front of a half-plane crack in a loaded elastic full space. That work also indicated the relation of such calculations to a three-dimensional weight function theory for crack analysis and derived an expression for the distribution of the tensile mode stress intensity factor along a slightly curved crack front, to first order accuracy in the deviation of the crack front location from a reference straight line. Here we extend the results on stress intensity factors to the shear modes, solving to similar first order accuracy for the in-plane (Mode 2) and antiplane (Mode 3) shear stress intensity factors along a slightly curved crack front. Implications of results for the configurational stability of a straight crack front are discussed. It is also shown that the concept of line tension, while qualitatively useful in characterizing the crack extension force (energy release rate) distribution exerted on a tough heterogeneity along a fracture path as the crack front begins to curve around it, does not agree with the exact first order effect that is derived here.

\section{Introduction}

For a half-plane crack lying in an infinite space, the stress intensity factors due to point force pairs acting on the crack surface have been derived by many authors (Uflyand, 1965; Sih and Liebowitz, 1968; Kassir and Sih, 1973; Bueckner, 1977; Meade and Keer, 1984a; etc) in the case when the crack front lies along a straight line. Hence, by integration, the solution due to arbitrary loading on the crack surface can be found.

Rice (1985a) showed how the knowledge of such solutions enables one to calculate the changes in crack surface displacement distribution, exact to the first order in the deviation of the crack front position from a reference straight line, when the crack front position is altered slightly to lie along a general curved arc in the same plane as that of the crack. He gave full details for the case of tensile (Mode 1) loading and derived an expression for the stress intensity factor $K_{1}$ along such a nonstraight crack front (again, exact to the first order). The latter work was motivated by the interesting approach to the wavy crack front problem based on asymptotic expansions by Meade and Keer (1984b), although it turned out that their results required correction.

Contributed by the Applied Mechanics Division for presentation at the Winter Annual Meeting, Anaheim, CA, December 7-12, 1986, of The American Society of Mechanical Engineers.

Discussion on this paper should be addressed to the Editorial Department, ASME, United Engineering Center, 345 East 47th Street, New York, N.Y. 10017 , and will be accepted until two months after final publication of the paper itself in the Journal of APPLIEd MEchanics. Manuscript received by ASME Applied Mechanics Division, September 20, 1985; final revision, April 14, 1986. Paper No. 86-WA/APM-43.
Here we carry through details of the slightly curved crack front analysis for general shear loading, deriving the analogous expressions for the inplane (Mode 2) and antiplane (Mode 3) stress intensity factors, $K_{2}$ and $K_{3}$, along a nonstraight crack front.

\section{Crack Surface Displacement}

We now present the basic equation for crack surface displacements associated with incremental crack growth, following Rice (1985a).

An infinite, homogeneous, isotropic elastic solid contains a half-plane crack with a straight crack front and is subjected to an "original" load system, consisting of some fixed forces and/or imposed boundary displacements, that induces mixed modes with distributions $K_{\alpha}^{0}\left(z^{\prime}\right)$ of stress intensity factors along the crack front. Here $\alpha=1,2,3$ and $z^{\prime}$ denotes the location along the crack front. A Cartesian $x, y, z$ coordinate system is attached such that the crack plane lies on $y=0$ and the $z$ axis lies along the crack front (Fig. 1).

Now imagine that the original loading is supplemented by a set of concentrated force pairs $\pm P_{j}, j=x, y, z$, acting at $x$, $0^{+}, z$ and $x, 0^{-}, z$ resulting in opening, inplane shear and antiplane shear relative displacements of the crack surface. Let $\Delta u_{j}(x, z)$ be the relative displacements of crack surfaces at the load location. (These are unbounded for point forces; see Rice (1985a) for a refinement of the argument by distributing the forces over finite discs whose radius is later allowed to approach zero.) Suppose that under the combined load system described, the crack front is advanced normal to itself by some infinitesimal variable distance $\delta a\left(z^{\prime}\right)$, where $z^{\prime}$ is the location 


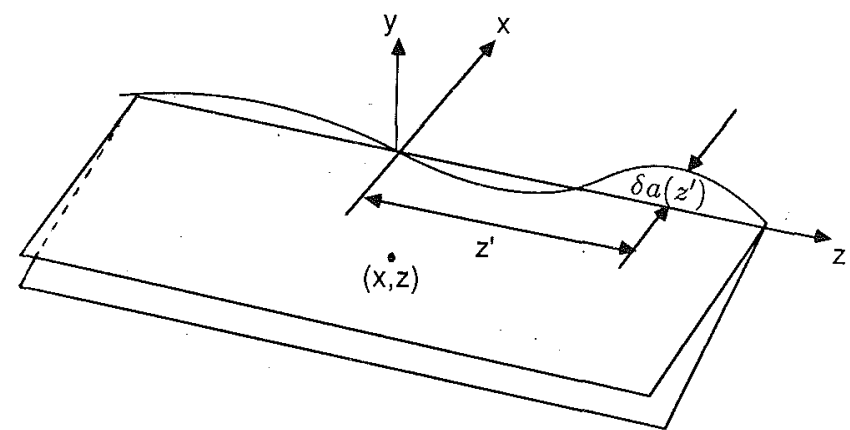

Fig. 1 Half-plane crack on $y=0$ in an infinite elastic body. Reference straight crack front along $z$ axis; $z$ ' denotes location along front and $\delta a\left(z^{\prime}\right)$ denoles advance of crack location in the plane $y=0$

along the front. The change in strain energy $U$ plus the potential energy $V_{0}$ of the fixed forces of the "original load system", associated with incremental crack growth and/or variation of the point force amplitudes $P_{j}$, is

$$
\delta\left(U+V_{0}\right)=P_{j} \delta\left[\Delta u_{j}(x, z)\right]-\int_{-\infty}^{+\infty} G\left(z^{\prime}\right) \delta a\left(z^{\prime}\right) d z^{\prime}
$$

$(j=x, y, z ;$ summation implied on repeated $j$.

Here $G$ is the energy release rate per unit crack area of elastic fracture mechanics, and by Irwin's relation

$$
G=\left(1-\nu^{2}\right)\left(K_{1}^{2}+K_{2}^{2}\right) / E+(1+\nu) K_{3}^{2} / E
$$

( $E=$ Young's modulus, $\nu=$ Poisson's ratio.)

Since $U$ and $V_{0}$ can depend only on the $P_{j}$ (or $\Delta u_{j}$ ) and the location of the crack front, the right-hand side of equation (1) is a perfect differential. Rice (1985a) then showed, using a Legendre transformation and the reciprocal property of coefficients in perfect differentials, that the variation of surface displacemnets due to incremental crack front advance under fixed applied forces is

$$
\begin{array}{r}
\delta\left[\Delta u_{j}(x, z)\right]=\int_{-\infty}^{+\infty} \frac{\partial G\left(P_{j} ; z^{\prime}\right)}{\partial P_{j}} \delta a\left(z^{\prime}\right) d z^{\prime} \\
=\int_{-\infty}^{+\infty}\left(2 \frac { 1 - \nu ^ { 2 } } { E } \left[K_{1}\left(P_{j} ; z^{\prime}\right) \frac{\partial K_{1}\left(P_{j} ; z^{\prime}\right)}{\partial P_{j}}\right.\right. \\
\left.+K_{2}\left(P_{j} ; z^{\prime}\right) \frac{\partial K_{2}\left(P_{j} ; z^{\prime}\right)}{\partial P_{j}}\right] \\
\left.+2 \frac{1+\nu}{E} K_{3}\left(P_{j} ; z^{\prime}\right) \frac{\partial K_{3}\left(P_{j} ; z^{\prime}\right)}{\partial P_{j}}\right) \delta a\left(z^{\prime}\right) d z^{\prime}
\end{array}
$$

Here the derivations of $G$ and the $K_{\alpha}$ with respect to the $P_{j}$ are taken with a fixed position of the crack front. Letting the $P_{j}$ $=0$, one has

$$
\begin{gathered}
K_{\alpha}\left(0 ; z^{\prime}\right)=K_{\alpha}^{0}\left(z^{\prime}\right) \\
\partial K_{\alpha}\left(0 ; z^{\prime}\right) / \partial P_{j}=k_{\alpha j}\left(z^{\prime} ; x, z\right)
\end{gathered}
$$

$(\alpha=1,2,3, j=x, y, z)$ where $K_{\alpha}^{0}\left(z^{\prime}\right)$ is the mode $\alpha$ stress intensity factor induced along the reference straight crack front by the original loading while $k_{\alpha j}$ is the mode $\alpha$ stress intensity factor induced by a unit force pair at $\left(x, 0^{+}, z\right)$ and $\left(x, 0^{-}, z\right)$ in the $\pm j$ directions. Thus equation (3) becomes

$$
\begin{gathered}
\delta\left[\Delta u_{j}(x, z)\right]=\int_{-\infty}^{+\infty}\left(\frac { 2 ( 1 - \nu ^ { 2 } ) } { E } \left[k_{1 j}\left(z^{\prime} ; x, z\right) K_{1}^{0}\left(z^{\prime}\right)\right.\right. \\
\left.+k_{2 j}\left(z^{\prime} ; x, z\right) K_{2}^{0}\left(z^{\prime}\right)\right] \\
\left.+\frac{2(1+\nu)}{E} k_{3 j}\left(z^{\prime} ; x, z\right) K_{3}^{0}\left(z^{\prime}\right)\right) \delta a\left(z^{\prime}\right) d z^{\prime}
\end{gathered}
$$

Equation (4) is the first order variation of $\Delta u_{j}(x, z)$ when the crack advances by $\delta a\left(z^{\prime}\right)$ in presence of the original load system only. In fact, equation (4) can be regarded as a special version of a general three-dimensional relation in Rice's (1972) formulation (see Rice $1985 \mathrm{a}, \mathrm{b}$ also), based on displacement field variations associated with incremental crack growth, of the theory of Bueckner's (1970, 1972, 1977) "weight functions" for crack analysis.

As we stated before, $k_{\alpha j}$ can be found for the present halfplane crack configuration from many sources in the literature, and they are also listed in the form

$$
\begin{gathered}
k_{1 x}=k_{1 z}=k_{2 y}=k_{3 y}=0 \\
k_{1 y}=k=\frac{\left(-2 x / \pi^{3}\right)^{1 / 2}}{\left[x^{2}+\left(z^{\prime}-z\right)^{2}\right]} \\
k_{2 x}=\left[1+\frac{2 \nu}{2-\nu} \frac{x^{2}-\left(z^{\prime}-z\right)^{2}}{x^{2}+\left(z^{\prime}-z\right)^{2}}\right] k \\
k_{3 z}=\left[1-\frac{2 \nu}{2-\nu} \frac{x^{2}-\left(z^{\prime}-z\right)^{2}}{x^{2}+\left(z^{\prime}-z\right)^{2}}\right] k \\
k_{2 z}=k_{3 x}=-\frac{4 \nu}{2-\nu} \frac{x\left(z^{\prime}-z\right)}{x^{2}+\left(z^{\prime}-z\right)^{2}} k
\end{gathered}
$$

by Rice (1985a).

\section{Shear Mode Stress Intensity Factors}

Substituting equations (5) into equation (4), for the shear displacements we obtain

$$
\begin{gathered}
\delta\left[\Delta u_{x}(x, z)\right]=\frac{8\left(1-\nu^{2}\right)}{E}\left(-\frac{x}{2 \pi}\right)^{1 / 2}\left[\frac{1}{2 \pi} \int_{-\infty}^{+\infty} \frac{K_{2}^{0}\left(z^{\prime}\right)}{x^{2}+\left(z^{\prime}-z\right)^{2}}\right. \\
\left(1+\frac{2 \nu}{2-\nu} \frac{x^{2}-\left(z^{\prime}-z\right)^{2}}{x^{2}+\left(z^{\prime}-z\right)^{2}}\right) \delta a\left(z^{\prime}\right) d z^{\prime} \\
\left.-\frac{2 \nu}{\pi(2-\nu)(1-\nu)} \int_{-\infty}^{+\infty} \frac{K_{3}^{0}\left(z^{\prime}\right) x\left(z^{\prime}-z\right)}{\left[x^{2}+\left(z^{\prime}-z\right)^{2}\right]^{2}} \delta a\left(z^{\prime}\right) d z^{\prime}\right] \\
\delta\left[\Delta u_{z}(x, z)\right]=\frac{8(1+\nu)}{E}\left(-\frac{x}{2 \pi}\right)^{1 / 2}\left[\frac{1}{2 \pi} \int_{-\infty}^{+\infty} \frac{K_{3}^{0}\left(z^{\prime}\right)}{x^{2}+\left(z^{\prime}-z\right)^{2}}\right. \\
\left(1-\frac{2 \nu}{2-\nu} \frac{x^{2}-\left(z^{\prime}-z\right)^{2}}{x^{2}+\left(z^{\prime}-z\right)^{2}}\right) \delta a\left(z^{\prime}\right) d z^{\prime} \\
\left.-\frac{2 \nu(1-\nu)}{\pi(2-\nu)} \int_{-\infty}^{+\infty} \frac{K_{2}^{0}\left(z^{\prime}\right) x\left(z^{\prime}-z\right)}{\left[x^{2}+\left(z^{\prime}-z\right)^{2}\right]^{2}} \delta a\left(z^{\prime}\right) d z^{\prime}\right]
\end{gathered}
$$

correct to first order in $\delta a\left(z^{\prime}\right)$.

Very near the crack front the stress intensity factors are related to the relative displacements by the asymptotic formulae

$$
\begin{aligned}
& \Delta u_{n}(x, z)=\frac{8\left(1-\nu^{2}\right)}{E}\left(\frac{r}{2 \pi}\right)^{1 / 2} K_{2}+0\left[(r)^{3 / 2}\right] \\
& \Delta u_{t}(x, z)=\frac{8(1+\nu)}{E}\left(\frac{r}{2 \pi}\right)^{1 / 2} K_{3}+0\left[(r)^{3 / 2}\right]
\end{aligned}
$$

where $n$ and $t$ are the normal and tangential directions along the curved crack front (Fig. 2), with $n$ lying in the $x, z$ plane, and $r$ is the distance as measured from the crack front in the negative normal direction (Fig. 2). Denoting the angle between the normal and the $x$ direction (very small) by $\phi$, we find from geometric relations that

$$
-r \cos \phi=x-\delta a(z), \tan \phi=\mathrm{d}[\delta a(z)] / d z
$$

Now consider a particular $z$ at which $\delta a(z)=0$ but $d[\delta a(z)] / d z$ exists. Then the first of equations (6) becomes, when $x \rightarrow 0^{-}$,

$$
\delta\left[\Delta u_{x}(x, z)\right] \sim \frac{8\left(1-\nu^{2}\right)}{E}\left(-\frac{x}{2 \pi}\right)^{1 / 2}[\ldots]
$$




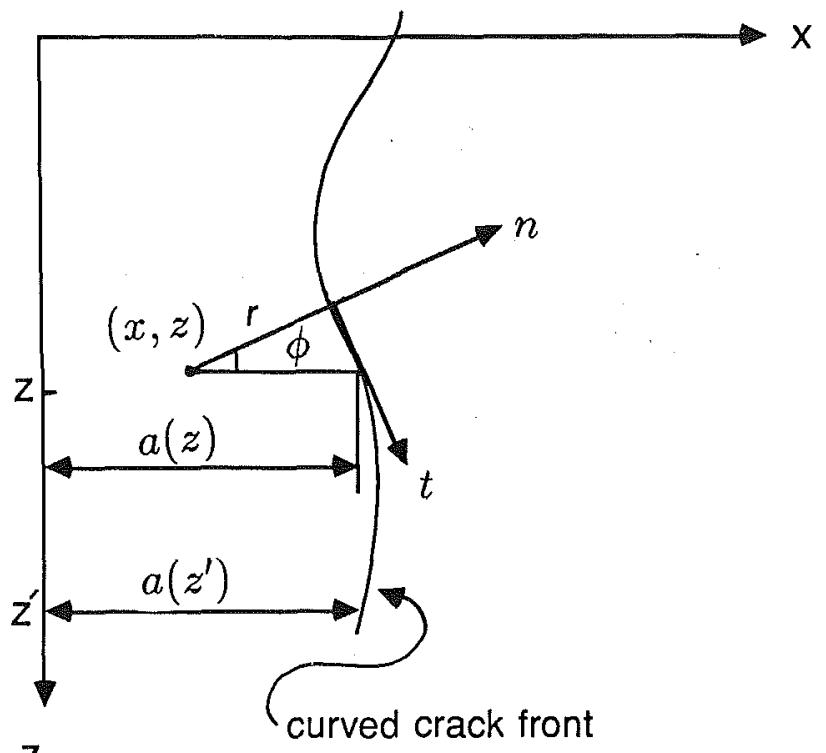

Z

Fig. 2 Normal (n) and tangential (t) directions for deviated half-plane crack

where now the bracketed terms of equation (6) have reduced to

$$
\begin{aligned}
{[\ldots]=\frac{1}{2 \pi} } & \frac{2-3 \nu}{2-\nu} P V \int_{-\infty}^{+\infty} \frac{K_{2}^{0}\left(z^{\prime}\right) \delta a\left(z^{\prime}\right)}{\left(z^{\prime}-z\right)^{2}} d z^{\prime} \\
& +\frac{\nu}{(2-\nu)(1-\nu)} K_{3}^{0}(z) \frac{d[\delta a(z)]}{d z}
\end{aligned}
$$

and where the $P V$ in the first term of equation (9) denotes the principal value in the Cauchy sense.

Equation (9) can be proven as follows. Breaking the $\int_{-\infty}^{+\infty}$ in the first of equation (6) into $\int_{-\infty}^{z-\eta}+\int_{z-\eta}^{z+\eta}+\int_{z+\eta}^{+\infty}$, the $\left[\int_{-\infty}^{z-\eta}+\right.$ $\left.\int_{z+\eta}^{+\infty}\right]$ part, when letting $x \rightarrow 0^{-}$and then $\eta \rightarrow 0$, gives the $P V$ term in (9) above, whereas the remaining $\int_{z-\eta}^{z+\eta}$ part of the bracketed term in equation (6) becomes

$$
\begin{gathered}
\frac{1}{2 \pi} \int_{z-\eta}^{z+\eta} \frac{K_{2}^{0}\left(z^{\prime}\right)}{x^{2}+\left(z^{\prime}-z\right)^{2}}\left[1+\frac{2 \nu}{2-\nu} \frac{x^{2}-\left(z^{\prime}-z\right)^{2}}{x^{2}+\left(z^{\prime}-z\right)^{2}}\right] \delta a\left(z^{\prime}\right) d z^{\prime} \\
-\frac{2 \nu}{\pi(2-\nu)(1-\nu)} \int_{z-\eta}^{z+\eta} \frac{K_{3}^{0}\left(z^{\prime}\right) x\left(z^{\prime}-z\right)}{\left[x^{2}+\left(z^{\prime}-z\right)^{2}\right]^{2}} \delta a\left(z^{\prime}\right) d z^{\prime}
\end{gathered}
$$

Let us now observe that

$$
K_{\alpha}^{0}\left(z^{\prime}\right) \delta a\left(z^{\prime}\right)=K_{\alpha}^{0}(z) \frac{d[\delta a(z)]}{d z}\left(z^{\prime}-z\right)+O\left[\left(z^{\prime}-z\right)^{2}\right]
$$

and that the error term $O\left[\left(z^{\prime}-z\right)^{2}\right]$ will have a bound of form $\left|O\left[\left(z^{\prime}-z\right)^{2}\right]\right| \leq B\left(z^{\prime}-z\right)^{2}$ on $z-\eta \leq z^{\prime} \leq z+\eta$ for some finite $B>0$. Then since the term linear in $\left(z^{\prime}-z\right)$ gives zero contribution to the first integral above, i,e., to the integral involving $K_{2}^{0}$, we have

$$
\begin{aligned}
& \left|\frac{1}{2 \pi} \int_{z-\eta}^{z+\eta} \frac{K_{2}^{0}\left(z^{\prime}\right)}{x^{2}+\left(z^{\prime}-z\right)^{2}}\left[1+\frac{2 \nu}{2-\nu} \frac{x^{2}-\left(z^{\prime}-z\right)^{2}}{x^{2}+\left(z^{\prime}-z\right)^{2}}\right] \delta a\left(z^{\prime}\right) d z^{\prime}\right| \\
& \leq \frac{B}{2 \pi} \int_{z-\eta}^{z+\eta} \frac{\left(z^{\prime}-z\right)^{2}}{x^{2}+\left(z^{\prime}-z\right)^{2}}\left[1+\frac{2 \nu}{2-\nu}\right] d z^{\prime}<\frac{2+\nu}{2-\nu} \frac{B}{\pi} \eta
\end{aligned}
$$

for any $x$. Hence, letting $x \rightarrow 0^{-}$and then $\eta \rightarrow 0$, the upper bound on the first integral, and therefore the integral itself, vanishes. In the same limit the second integral, involving $K_{3}^{0}$, becomes with the substitution $z^{\prime}-z=-t x$

$$
\begin{array}{r}
\frac{2 \nu}{\pi(2-\nu)(1-\nu)} \int_{-\infty}^{+\infty} \frac{t^{2}}{\left(1+t^{2}\right)^{2}} d t K_{3}^{0}(z) \frac{d[\delta a(z)]}{d z} \\
=\frac{\nu}{(2-\nu)(1-\nu)} K_{3}^{0}(z) \frac{d[\delta a(z)]}{d z}
\end{array}
$$

We thus have the two contributions noted in equation (9), the $P V$ term from $\left[\int_{-\infty}^{z-\eta}+\int_{z+\eta}^{+\infty}\right]\left(\eta \rightarrow 0^{+}\right)$, which represents the influence of the rest of the nonstraight crack front on the special point $z$ at which $\delta a(z)=0$, and the term involving $K_{3}^{0}(z)$ from $\int_{z-\eta}^{z+\eta}$ which represents the coupling effect due to local slope. The sum of these two is given as equation (9). In the above argument we have implicitly assumed, in writing the error terms as $O\left[\left(z^{\prime}-z\right)^{2}\right]$, that $K_{\alpha}^{0}\left(z^{\prime}\right) \delta a\left(z^{\prime}\right)$ has a good second derivative at $z$. However, the steps leading to equation (9) above, and equation (10) below, may be justified under weaker assumption that the first derivative of $K_{\alpha}^{0}\left(z^{\prime}\right) \delta a\left(z^{\prime}\right)$ exists and is merely Holder continuous at $z$, such that the bounded term above may be written as $B \mid z^{\prime}-z^{1+\varepsilon}$ where 0 $<\epsilon \leq 1$.

Similary, when $x \rightarrow 0^{-}$the second of equation (6) reduces to

$$
\delta\left[\Delta u_{z}(x, z)\right] \sim \frac{1+\nu}{E}\left(-\frac{x}{2 \pi}\right)^{1 / 2}[\ldots]
$$

where now

$$
\begin{gathered}
{[\ldots]=\frac{1}{2 \pi} \frac{2+\nu}{2-\nu} P V \int_{-\infty}^{+\infty} \frac{K_{3}^{0}\left(z^{\prime}\right) \delta a\left(z^{\prime}\right)}{\left(z^{\prime}-z\right)^{2}} d z^{\prime}} \\
+\frac{\nu(1-\nu)}{2-\nu} K_{2}^{0}(z) \frac{d[\delta a(z)]}{d z}
\end{gathered}
$$

in which we can also observe the coupling due to local slope.

To find $\delta\left[\Delta u_{x}(x, z)\right]$ and $\delta\left[\Delta u_{z}(x, z)\right]$ near some location $z$ along the crack front where $\delta a(z) \neq 0$, we use the concept developed by Rice (1985a) of relocating the reference straight crack front by moving it along the $x$ direction an amount equal to $\delta a(z)$. Then, redefining the origin of the $x$ axis so that $x=$ 0 along the relocated reference straight crack tip, we have $\delta a(z)=0$ at the location $z$ considered and can use the results just given above. Note that the reference straight crack location is aribtrary, so that we can locate it at will. In other words we always choose the reference straight crack as the one that when $x \rightarrow 0^{-}$we approach simultaneously both the reference straight crack and the actual front. This is equivalent to interpreting $\delta a\left(z^{\prime}\right)$ in the above formulae as $a\left(z^{\prime}\right)-a(z)$, where $x$ $=a(z)$ is the slightly curved arc describing the crack front location relative to any convenient choice of origin for the $x$ axis, and interpreting $d[\delta a(z)] / d z$ as $d a(z) / d z$. In using this notation one also needs expressions for the stress intensity factors induced at location $z$ along the crack front when the front is straight but located at a general $x$ coordinate, say, $x=a$. We let the functions $K_{\alpha}^{0}[z ; a], \alpha=1,2,3$, denote these distributions. Then, at a given point $x, z$ on the crack faces, equations (9) and (10) become

$$
\begin{gathered}
\delta\left[\Delta u_{x}(x, z)\right] \sim \frac{8\left(1-\nu^{2}\right)}{E}\left[\frac{a(z)-x}{2 \pi}\right]^{1 / 2} \times \\
\left\{\frac{1}{2 \pi} \frac{2-3 \nu}{2-\nu} P V \int_{-\infty}^{+\infty} \frac{K_{2}^{0}\left[z^{\prime} ; a(z)\right]\left[a\left(z^{\prime}\right)-a(z)\right]}{\left(z^{\prime}-z\right)^{2}} d z^{\prime}\right. \\
\left.+\frac{\nu}{(2-\nu)(1-\nu)} K_{3}^{0}[z ; a(z)] \frac{d a(z)}{d z}\right\}
\end{gathered}
$$

and

$$
\begin{gathered}
\delta\left[\Delta u_{z}(x, z)\right] \sim \frac{8(1+\nu)}{E}\left[\frac{a(z)-x}{2 \pi}\right]^{1 / 2} \times \\
\left\{\frac{1}{2 \pi} \frac{2+\nu}{2-\nu} P V \int_{-\infty}^{+\infty} \frac{K_{3}^{0}\left[z^{\prime} ; a(z)\right]\left[a\left(z^{\prime}\right)-a(z)\right]}{\left(z^{\prime}-z\right)^{2}} d z^{\prime}\right. \\
\left.+\frac{\nu(1-\nu)}{(2-\nu)} K_{2}^{0}[z ; a(z)] \frac{d a(z)}{d z}\right\}
\end{gathered}
$$

to first order in $a\left(z^{\prime}\right)-a(z)$ and in $d a(z) / d z$. These last equations are now understood to represent the change in sur- 
face displacement at a point $x, z$ very near crack front in going from the hypothetical reference state, in which the crack front is everywhere straight and of depth equal to that at $z$, to the actual state in which the crack front is curved.

Referring to Fig. 2, we can get the relative displacement components in the normal and tangential directions along the crack front in terms of $\Delta u_{z}(x, z)$ and $\Delta u_{x}(x, z)$,

$$
\begin{aligned}
& \Delta u_{n}(x, z)=\Delta u_{x}(x, z) \cos \phi-\Delta u_{z}(x, z) \sin \phi \\
& \Delta u_{t}(x, z)=\Delta u_{x}(x, z) \sin \phi+\Delta u_{z}(x, z) \cos \phi
\end{aligned}
$$

As $\phi$ is small for a small perturbation we have to the first order

$$
\begin{aligned}
& \Delta u_{n}(x, z)=\Delta u_{x}-\Delta u_{z} d a(z) / d z \\
& \Delta u_{t}(x, z)=\Delta u_{z}+\Delta u_{x} d a(z) / d z
\end{aligned}
$$

In equations (13) we now write $\Delta u_{x}$ as $\left(\Delta u_{x}\right)^{0}+\delta\left(\Delta u_{x}\right)$ and

the $x$ and $y$ dependence; in fact, just the opposite was found to be true in the solution developed (without $a$ priori assumptions on scaling) by Rice. Similar remarks apply to the Meade and Keer results for Modes 2 and 3.

The results of equations (15) for $K_{\alpha}(z)$ can be inserted into equation (2) to compute the energy release rate $G(z)$ along the slightly curved crack front. When this is done and we retain only those terms consistent with first order accuracy in $a\left(z^{\prime}\right)-a(z)$ and $d a(z) / d z$, we find that the cross terms involvig $d a(z) d z$ in equations (15) cancel one another. A specific illustration of this is given in the next section.

It may sometimes prove convenient to rewrite the various principal value integrals of equation (15) by the rearrangement, following from integration by parts,

$P V \int_{-\infty}^{+\infty} \frac{K_{\alpha}^{0}\left[z^{\prime} ; a(z)\right]\left[a\left(z^{\prime}\right)-a(z)\right]}{\left(z^{\prime}-z\right)^{2}} d z^{\prime}$

$$
=P V \int_{-\infty}^{+\infty} \frac{K_{\alpha}^{0}\left[z^{\prime} ; a(z)\right] d a\left(z^{\prime}\right) / d z^{\prime}+\left[a\left(z^{\prime}\right)-a(z)\right] \partial K_{\alpha}^{0}\left[z^{\prime} ; a(z)\right] / \partial z^{\prime}}{\left(z^{\prime}-z\right)} d z^{\prime}
$$

$\Delta u_{z}$ as $\left(\Delta u_{z}\right)^{0}+\delta\left(\Delta u_{z}\right)$, where $\left(\Delta u_{x}\right)^{0}$ and $\left(\Delta u_{z}\right)^{0}$ are the near-tip crack face displacements in the reference straight crack front configuration and, again, $\delta\left(\Delta u_{x}\right)$ and $\delta\left(\Delta u_{z}\right)$ are the variations of equation (11) due to the crack front being curved, i.e., due to the crack front advancing by $\delta a\left(z^{\prime}\right)=$ $a\left(z^{\prime}\right)-a(z)$ :

$$
\begin{aligned}
& \Delta u_{n}(x, z)=\left(\Delta u_{x}\right)^{0}+\delta\left[\Delta u_{x}\right]-\left(\Delta u_{z}\right)^{0} d a(z) / d z \\
& \Delta u_{i}(x, z)=\left(\Delta u_{z}\right)^{0}+\delta\left[\Delta u_{z}\right]+\left(\Delta u_{x}\right)^{0} d a(z) / d z
\end{aligned}
$$

Everything here is exact to first order in $\delta a(z)$. Comparing these expressions, as evaluated with the help of equations (11), to equations (7) (and recognizing that $r=a(z)-x$ to first order) we get the stress intensity factors $K_{2}$ and $K_{3}$ to first order when the crack front deviates from a reference straight line. The results, supplemented for completeness with the result for the Mode 1 stress intensity factor derived by Rice (1985a), are as follows:

$$
\begin{aligned}
K_{1}(z) & =K_{1}^{0}[z ; a(z)] \\
+ & \frac{1}{2 \pi} P V \int_{-\infty}^{+\infty} \frac{K_{1}^{0}\left[z^{\prime} ; a(z)\right]\left[a\left(z^{\prime}\right)-a(z)\right]}{\left(z^{\prime}-z\right)^{2}} d z^{\prime} \\
K_{2}(z) & =K_{2}^{0}[z ; a(z)]-\frac{2}{2-\nu} K_{3}^{0}[z ; a(z)] \frac{d a(z)}{d z} \\
+\frac{1}{2 \pi} & \frac{2-3 \nu}{2-\nu} P V \int_{-\infty}^{+\infty} \frac{K_{2}^{0}\left[z^{\prime} ; a(z)\right]\left[a\left(z^{\prime}\right)-a(z)\right]}{\left(z^{\prime}-z\right)^{2}} d z^{\prime} \\
K_{3}(z) & =K_{3}^{0}[z ; a(z)]+\frac{2(1-\nu)}{2-\nu} K_{2}^{0}[z ; a(z)] \frac{d a(z)}{d z} \\
+\frac{1}{2 \pi} & \frac{2+\nu}{2-\nu} P V \int_{-\infty}^{+\infty} \frac{K_{3}^{0}\left[z^{\prime} ; a(z)\right]\left[a\left(z^{\prime}\right)-a(z)\right]}{\left(z^{\prime}-z\right)^{2}} d z^{\prime}
\end{aligned}
$$

Equations (15) are not consistent with the stress intensity factors presented for this case by Meade and Keer (1984b) as

$$
\begin{aligned}
& K_{1}(z)=K_{1}^{0}[z ; a(z)] \\
& K_{2}(z)=K_{2}^{0}[z ; a(z)]-K_{3}^{0}[z ; a(z)] d a(z) / d z \\
& K_{3}(z)=K_{3}^{0}[z ; a(z)]+K_{2}^{0}[z ; a(z)] d a(z) / d z
\end{aligned}
$$

Rice (1985a, just after his equation (65)), explained the source of oversight in the Meade and Keer results for Mode 1, in that Meade and Keer assumed in their asymptotic analysis a double scaling of the $z$ dependence of the solution but not of
The last term of the second numerator vanishes when, as in the next section, we consider loadings which would induce uniform $K_{\alpha}$ along a straight crack front.

\section{Cosine-Wave Crack Front; Configurational Stability}

Now we apply the results in equations (15) to the case of a wavy crack front with the profile

$$
a(z)=a_{0}+A \cos (2 \pi z / \lambda)
$$

where $A / \lambda<<1, a_{0}$ is arbitrary and $\lambda>0$. It is assumed for convenience that the stress intensity factors induced along the reference straight crack are uniform along the crack front i.e., $K_{\alpha}^{0}[z ; a]=K_{\alpha}^{0}[a]$. Going through some algebraic calculations and further assuming that $\left(A d K^{0} / d a\right) / K^{0}<<1$, we get the results to the first order in $A$

$$
\begin{gathered}
K_{1}(z)=K_{1}\left[a_{0}\right]+\left[\frac{d K_{1}^{0}\left[a_{0}\right]}{d a_{0}}-\pi K_{1}^{0}\left[a_{0}\right] / \lambda\right] A \cos \left(\frac{2 \pi z}{\lambda}\right) \\
K_{2}(z)=K_{2}\left[a_{0}\right]+\left[\frac{d K_{2}^{0}\left[a_{0}\right]}{d a_{0}}-\frac{2-3 \nu}{2-\nu} \pi K_{2}^{0}\left[a_{0}\right] / \lambda\right] A \cos \left(\frac{2 \pi z}{\lambda}\right) \\
+\frac{4 \pi}{2-\nu}(A / \lambda) K_{3}^{0}\left[a_{0}\right] \sin \left(\frac{2 \pi z}{\lambda}\right) \\
K_{3}(z)=K_{3}\left[a_{0}\right]+\left[\frac{d K_{3}^{0}\left[a_{0}\right]}{d a_{0}}-\frac{2+\nu}{2-\nu} \pi K_{3}^{0}\left[a_{0}\right] / \lambda\right] A \cos \left(\frac{2 \pi z}{\lambda}\right) \\
-\frac{4 \pi(1-\nu)}{2-\nu} K_{2}^{0}\left[a_{0}\right](A / \lambda) \sin \left(\frac{2 \pi z}{\lambda}\right)
\end{gathered}
$$

From equations (18) we observe that when both shear modes are present the extremal values of a given shear stress intensity factor do not occur at the extremal locations of the crack front where $\cos (2 \pi z / \lambda)= \pm 1$.

We may also compute the energy release rate $G$, defined by equation (2), along the perturbed crack front from the results just given for the $K_{\alpha}$. Consistent with the first order accuracy in $A$, the result is

$$
G(z)=G^{0}\left[a_{0}\right]+\left\{d G^{0}\left[a_{0}\right] / d a_{0}-2 \pi F\left[a_{0}\right] / \lambda\right\} A \cos (2 \pi z / \lambda)
$$

Here

$$
G^{0}\left[a_{0}\right]=\frac{1-\nu^{2}}{E}\left\{\left(K_{1}^{0}\left[a_{0}\right]\right)^{2}+\left(K_{2}^{0}\left[a_{0}\right]\right)^{2}+\frac{1}{1-\nu}\left(K_{3}^{0}\left[a_{0}\right]\right)^{2}\right\}
$$




$$
\begin{aligned}
F\left[a_{0}\right]=\frac{1-\nu^{2}}{E} & \left\{\left(K_{1}^{0}\left[a_{0}\right]\right)^{2}+\frac{2-3 \nu}{2-\nu}\left(K_{2}^{0}\left[a_{0}\right]\right)^{2}\right. \\
& \left.+\frac{2+\nu}{(2-\nu)(1-\nu)}\left(K_{3}^{0}\left[a_{0}\right]\right)^{2}\right\}
\end{aligned}
$$

Note that the extrema of $G(z)$ do coincide with the extrema of $a(z)$ since the sine terms of equation (18) have cancelled one another.

We have not been able to find an energetic interpretation for $F$ in equation (21) but we can confirm that the result derived for $G(z)$ in equation (19) is not compatible with a stringlike model with a "line tension" effect, as used frequently to analyze curved dislocation lines. In particular, $G$ is sometimes called the "crack extension force" since it is the generalized force, in the sense of equation (1), conjugate to crack growth. One might optimistically hope that the effect of curvature on the crack extension force would be analogous to the effect of curvature on a string under tension. Such would form a useful conceptual picture of, e.g., the crack extension force distribution exerted as a crack front meets and begins to surround a localized, hard-to-fracture heterogeneity lying in the path of the advancing crack. However, while the string model is qualitatively correct in predicting the proper sign of the effect of curvature shown in equation (19), it fails quantitatively since elementary calculations show that a line tension model would require a $1 / \lambda^{2}$ effect for a cosine wave rather than the proper $1 / \lambda$ effect that we have derived.

Rice (1985a) considered the configurational stability of quasi-static tensile mode crack growth (e.g., by fatigue or corrosion) by observing that if the crack growth rate is an increasing function of $K_{1}$, then the amplitude of a cosine component of $a(z)$ will grow if the maxima of $K_{1}(z)$ and $a(z)$ are in phase but decay if they are out of phase. Thus, from the first of equations (18), disturbances of wavelength $\lambda$ in the crack front profile will decay in amplitude during crack growth if

$$
d K_{1}^{0}\left[a_{0}\right] / d a_{0}<\pi K_{1}^{0}\left[a_{0}\right] / \lambda
$$

This is met for all $\lambda$ when $d K_{1}^{0} / d a_{0}<0$ and will be met for sufficiently small $\lambda$ when $d K_{1}^{0} / d a_{0}>0$. It generally turns out that the critical $\lambda$ values, $\lambda_{c \tau}$, at which the inequality fails (e.g., $\lambda_{c \tau}=2 \pi L$ for an edge crack of depth $L$ in a large body under remote tensile loading) are sufficiently large that the model of a half plane crack in an infinite body is inappropriate to analyze perturbations of those wavelengths; the actual finite body dimensions must be considered instead for a suitable analysis. Thus we conclude that planar crack growth should be configurationally stable to perturbations involving wavelengths that are small compared to overall body or crack dimensions. This seems to be generally in accord with experience in that cracks, when approximately planar, in subcritical growth are generally observed to have fronts that lie along smoothly curving arcs at the macroscale and to be devoid of structure except for that directly relatable to microstructure heterogeneity or large scale plastic flow. See, e.g., Colangelo and Heiser (1974, chapter 4).

The stability issue is less readily addressed under general mixed-mode loadings as we have analyzed them here since a mixed-mode crack will seldom grow along a plane. One case which may meet that condition of planarity involves the tectonic shear crack whose slip surface is channeled by a preexisting fault plane. If in that case or others it is approriate to describe crack advance under mixed-mode loading by a unique (independent of mode combination) increasing relation be- tween $G$ and the crack growth rate, then it is evident by comparing equations (19) to the first of equation (18) that a similar stability condition to that for Mode 1 growth will result. In particular, crack position $a(z)$ and $G(z)$ will be out of phase, thus smoothing out initial irregularities during growth, for wavelengths $\lambda$ satisfying

$$
d G^{0}\left[a_{0}\right] / d a_{0}<2 \pi F\left[a_{0}\right] / \lambda
$$

This reduces to equation (22) for pure Mode 1 conditions.

Meade and Keer (1984b) emphasized that crack front segmentation is observed in laboratory study of brittle materials under combined Mode 1 and Mode 3 loading. They suggest that this may be attributed to the coupling effect between the shear modes. For example, as is evident from the exact first order results in equations (15), or in equations (18) for the cosine wave, and as is also seen in the less complete Meade and Keer results reported here as equations (16), Mode 3 loading induces a Mode 2 stress intensity wherever $d a(z) / d z$ $\neq 0$. This induced $K_{2}$ reverses sign with the change in sign of $d a(z) / d z$ in going from one side to the other of a localized protrusion. This change in sign of $K_{2}$ is expected to promote deviations from planarity of opposite sense (up versus down relative to the $y$ direction) on the two sides of the protrusions during tensile crack growth, so that localized protrusions of the crack front grow into nonplanar segments. It is not yet clear how to test this proposed mechanism against observations.

\section{Acknowledgments}

The work reported was supported by the ONR Mechanics Division contract N00014-85-K-0405, USGS Earthquake Hazards Reduction Program grant 14-08-0001-G1167, and NSF Earth Sciences Division grant EAR-84-11201.

\section{References}

Bueckner, H. F., 1970, "A Novel Principle for the Computation of Stress Intensity Factors," Zeitschrift fuer angewandte Mathematik und Mechanik, Vol. 50 , pp. 529-546.

Bueckner, H. F., 1972, "Field Singularities and Related Integral Representations," Mechanics of Fracture 1: Methods of Analysis and Solution of Crack Problems, Sih, G. C., ed., Noordhoff, Leyden, pp. 329-314.

Bueckner, H. F., 1977, "The Weight Functions of Mode I of the PennyShaped and of the Elliptic Crack," Fracture Mechanics and Technology, Sih, G.

C., and Chow, eds., Vol. II, pp. 1069-1107, Sijthoff and Noordhoff publishers.

Colangelo, V. J., and Heiser, F. A., 1974, Analysis of Metallurgical Failures, Wiley, New York.

Kassir, M. K., and Sih, G. C., 1973, "Application of Papkovich-Neuber Potentials to a Crack Problem," International Journal of Solids and Structures, Vol. 9, pp. 643-645.

Meade, K. P., and Keer, L, M., 1984a, "On the Problem of a Pair of Point Forces Applied to the Faces of a Semi-Infinite Plane Crack," Journal of Elasticity, Vol. 14, pp. 3-14.

Meade, K. P., and Keer, L. M., 1984b, "Stress Intensity Factors for Semiinfinite Plane Crack with a Wavy Front," Journal of Elasticity, Vol, 14, pp. 79-92.

Rice, J. R., 1972, "Some Remarks on Elastic Crack Tip Stress Fields," International Journal of Solids and Structures, Vol. 8, pp. 751-758.

Rice, J. R., 1985a, "First Order Variations in Elastic Fields Due to Variation in Location of a Planar Crack Front," ASME Journal of Applied Mechanics, Vol. 52, pp. 571-579.

Rice, J. R., 1985b, "Three Dimensional Elastic Crack Tip Interactions with Transformation Strains and Dislocations," International Journal of Solids and Structures, Vol. 21, pp. 781-791.

Sih, G. C., and Liebowitz, H., 1968, "Mathematical Theories of Brittle Fracture," Treatise on Fracture, Liebowitz, H., ed., Vol. II, Academic Press, New York, pp. 67-190.

Uflyand, Y. S., 1965, "Survey of Articles on the Application of Integral Transforms in the Theory of Elasticity," North Carolina State University, Department of Applied Mathematics Research Group, File No. PSR-24/6, Raleigh, N.C. 